\title{
REALISMO NEOCLÁSICO: ¿EL RETORNO DE LOS ESTUDIOS INTERNACIONALES A LA CIENCIA POLÍTICA?*
}

\author{
Neoclassical Realism: The Return of International Studies \\ to Political Science?
}

\author{
VÍCTOR M. MIJARES \\ Universidad Simón Bolívar / German Institute of Global and Area Studies
}

\begin{abstract}
RESUMEN
Los estudios internacionales han alcanzado un importante grado de autonomía respecto a la ciencia política, y aquello lo demuestra su desarrollo en las principales universidades. Sin embargo, esta autonomía práctica dentro de las ciencias sociales tiene problemas para justificarse científicamente y cuenta además con un argumento débil en relación con el objeto de estudio. El auge del realismo neoclásico -fundada en la explicación de la política exterior y con base en la distribución del poder en el sistema internacional teniendo en cuenta la política nacional- tiene el potencial de revertir esa autonomía práctica al debilitar el argumento de esta. Inadvertidamente, el realismo neoclásico nos obligaría a revisar la autonomía de los estudios internacionales respecto a la ciencia política.
\end{abstract}

Palabras clave: Ciencia política, estudios internacionales, realismo neoclásico, teoría de las relaciones internacionales.

\begin{abstract}
International studies have reached a considerable degree of autonomy from political science. Evidence of this autonomy can be found in the emergence and development of international studies programs in leading universities. However, this autonomy within the social sciences is hardly ever justified in scientific terms. Moreover, arguments presented by international studies regarding their object of study are often weak. The paper argues that the rise of neoclassical realism, aiming at the explanation of foreign policy on the basis of the distribution of power (taking into account domestic politics), has the potential to reverse the autonomization of international studies. Perhaps inadvertently, neoclassical realism may invite us to revisit the autonomy of international studies and its relation with political science.
\end{abstract}

Key words: Political science, international studies, neoclassical realism, theory of international relations.

* Agradezco a Carlos A. Romero por sus observaciones y consejos al manuscrito original, a los revisores anónimos por la rigurosidad y el cuidado en sus labores, y al editor de RCP por la atención en todo el proceso de arbitraje. Cualquier error u omisión en el texto está bajo mi entera responsabilidad. 


\section{INTRODUCCIÓN}

La autonomía que en la práctica han venido alcanzando los estudios sobre relaciones internacionales (en adelante estudios internacionales o EE.II. ${ }^{1}$ ) presenta problemas para sostenerse como argumento en la medida en que su objeto de estudio no difiere del de la ciencia política (CP). El vigoroso desarrollo de teorías estructuralistas como el neorrealismo o el funcionalismo (ambas orientadas a considerar al sistema internacional como una estructura cuya lógica anárquica explica los grandes procesos políticos de conflicto y cooperación), así como la emergencia de teorías sociológicas (como la escuela inglesa o el constructivismo social, preocupadas fundamentalmente por buscar respuestas en las ideas e interpretaciones sobre la realidad, desestimando a la anarquía internacional como variable independiente), han reforzado la tesis de la independencia de los EE.II. respecto de otras ciencias sociales.

No obstante, el realismo neoclásico, corriente teórica emergente en los EE.II., plantea la refinación del realismo clásico y el neorrealismo. Asume que la distribución de capacidades en el sistema internacional (variable independiente) genera particulares tendencias en las políticas exteriores (variable dependiente), pero que estas varían de caso en caso en conformidad con las percepciones, capacidades y operatividad doméstica del "ejecutivo de política exterior" (EPE) (variable interviniente). La teoría del Estado, las distintas ramas del derecho público, la psicología, la geopolítica, la filosofía, la antropología y sobre todo la historia, vuelven a ocupar un lugar privilegiado en el análisis internacional como lo tuvieron en el realismo clásico. El resultado es un modelo y una agenda de investigación que permite abrir la caja negra del Estado y desplazar la imprecisa metáfora de las "bolas de billar" atribuida al neorrealismo, pero sin prescindir de sus valiosos principios analíticos de la política internacional y su propensión a la incorporación de métodos cuantitativos de operacionalización de variables. Estas cualidades nos permiten afirmar la potencialidad que tiene el realismo neoclásico para hacer evidente la unidad de lo político, con una potencial consecuencia de debilitar el argumento de la separación de los EE.II. y la CP, favoreciendo en el proceso a una posible reunificación disciplinaria plenamente aceptada. En este trabajo señalamos esa inadvertida posibilidad.

A continuación ofrecemos algunos datos y consideraciones sobre el grado de autonomía de la que gozan los EE.II. respecto de la CP. Luego exponemos los supuestos fundamentales del Realismo Neoclásico destacando los elementos que podrían favorecer la disposición a un retorno ampliamente aceptado de los EE.II. al campo politológico. Después destacamos el papel central del objeto de estudio en la unidad disciplinaria. En la sección final disertamos acerca de la plausibilidad y conveniencia de una aceptada reunificación disciplinaria plena.

1 A fin de evitar confusiones entre las relaciones internacionales como manifestación social interactiva más allá de las fronteras de un Estado, y las relaciones internacionales como esfuerzo por comprender dicha manifestación, preferimos usar el término "estudios internacionales" para el segundo caso y reservar el original para el primero.

2 FPE, por sus siglas en inglés. 


\section{AUTONOMÍA EN LA PRÁCTICA}

¿Son los EE.II. una disciplina autónoma o son un campo de estudio de la CP? Las implicaciones prácticas de la respuesta que se considere correcta han dado forma a departamentos y escuelas en universidades alrededor del mundo. Tomando como muestra las primeras 40 universidades (de un universo de 500) en el área de CP y EE.II. según el ranking Top Universities 2013 publicado por la empresa británica Quacquarelli Symonds (QS) (2014a), descubrimos que en poco más de la mitad de ellas (52\%) los EE.II. se imparten como una disciplina autónoma, mientras que en el resto forman parte de los programas de CP. En América Latina, de un universo de 300 universidades que otorgan títulos en CP y/o EE.II., una muestra ponderada de 36 (considerando la proporción del número de universidades por país en el ranking y una distribución que fuese geográficamente representativa), arrojó que un 58,5\% tienen programas de EE.II. autónomos respecto a los de $\mathrm{CP}{ }^{3}$

Tabla 1. Los estudios internacionales y la ciencia política en las principales universidades del mundo

\begin{tabular}{lcc}
\hline \multicolumn{1}{c}{ Universidad } & $\begin{array}{c}\text { EE.II. como } \\
\text { disciplina autónoma }\end{array}$ & $\begin{array}{c}\text { EE.II. } \\
\text { integrada a CP }\end{array}$ \\
\hline Harvard University & No & Sí \\
London School of Economics \& PolSci & Sí & No \\
Yale University & Sí & No \\
University of Oxford & No & Sí \\
Princeton University & Sí & No \\
Australian National University & Sí & No \\
University of Cambridge & No & Sí \\
Columbia University & Sí́ & No \\
The University of Tokyo & Sí & No \\
National University of Singapore & No & Sí \\
University of California, Berkeley & Sí & No \\
The University of Hong Kong & No & Sí \\
Kyoto University & No & Sí \\
New York University & Sí & No \\
Georgetown University & Sí & No \\
Sciences Po Paris & No & Sí \\
The University of Manchester & No & Sí \\
University of Toronto & Sí & No \\
University of California, San Diego & Sí & No \\
The University of Melbourne & No & Sí \\
Cornell University & No & Sí \\
Peking University & Sí & No \\
The University of Warwick & No & Sí \\
\hline
\end{tabular}




\begin{tabular}{lcc}
\hline \multicolumn{1}{c}{ Universidad } & $\begin{array}{c}\text { EE.II. como } \\
\text { disciplina autónoma }\end{array}$ & $\begin{array}{c}\text { EE.II. } \\
\text { integrada a CP }\end{array}$ \\
\hline Tsinghua University & Sí No \\
Fudan University & Sí & No \\
Stanford University & No & Sí \\
King's College London & Sí & No \\
The University of Sidney & Sí & No \\
University of California, Los Angeles & Sí & No \\
University of Michigan & Sí & No \\
Trinity College Dublin & No & Sí \\
The University of Chicago & No & Sí \\
The University of Edinburgh & No & Sí \\
The University of Queensland & Sí & No \\
Leiden University & Sí & No \\
Johns Hopkins University & Sí & No \\
Massachusetts Institute of Technology & No & Sí \\
McGill University & No & Sí \\
University of Copenhagen & No & Sí \\
The University of Auckland & No & Sí \\
\hline TOTAL & 21 & 19 \\
\hline$\%$ & 52,5 & 47,5 \\
\hline
\end{tabular}

Fuente: Elaboración propia a partir de los datos de los portales web del QS y de cada universidad.

\section{Tabla 2. Los estudios internacionales y la ciencia política en las principales universidades} latinoamericanas

\begin{tabular}{|c|c|c|c|}
\hline $\begin{array}{l}\text { País (número de } \\
\text { universidades } \\
\text { en el ranking } \\
\text { latinoamericano } \\
\text { 2013) }\end{array}$ & Universidad(es) de la muestra & $\begin{array}{l}\text { EE.II. como } \\
\text { disciplina } \\
\text { autónoma }\end{array}$ & $\begin{array}{l}\text { EE.II. } \\
\text { integrada } \\
\text { a CP }\end{array}$ \\
\hline \multirow{3}{*}{ Argentina (30) } & Universidad de Buenos Aires & No & Sí \\
\hline & $\begin{array}{l}\text { Pontificia Universidad Católica Argentina Santa } \\
\text { María de los Buenos Aires }\end{array}$ & Sí & No \\
\hline & Universidad de San Andrés & Sí & No \\
\hline \multirow[t]{5}{*}{ Bolivia (2) } & Universidad Mayor de San Andrés & No & Sí \\
\hline & Universidade de São Paulo & Sí & No \\
\hline & Universidade Estadual de Campinas & No & Sí \\
\hline & Universidade Federal do Rio de Janeiro & Sí & No \\
\hline & Universidade Federal de Minas Gerais & Sí & No \\
\hline \multirow[t]{4}{*}{ Brasil (81) } & $\begin{array}{l}\text { Universidade Estadual Paulista } \\
\text { "Julio Mesquita Filho" }\end{array}$ & Sí & No \\
\hline & Universidades Federal do Rio Grande Do Sul & Sí & No \\
\hline & Universidade Federal do São Paulo & Sí & No \\
\hline & Pontifícia Universidade Católica do Rio de Janeiro & Sí & No \\
\hline
\end{tabular}




\begin{tabular}{|c|c|c|c|}
\hline $\begin{array}{l}\text { País (número de } \\
\text { universidades } \\
\text { en el ranking } \\
\text { latinoamericano } \\
\text { 2013) }\end{array}$ & Universidad(es) de la muestra & $\begin{array}{c}\text { EE.II. como } \\
\text { disciplina } \\
\text { autónoma }\end{array}$ & $\begin{array}{c}\text { EE.II. } \\
\text { integrada } \\
\text { a CP }\end{array}$ \\
\hline \multirow{3}{*}{ Chile (30) } & Pontificia Universidad Católica de Chile & No & Sí \\
\hline & Universidad de Chile & Sí & No \\
\hline & Universidad de Santiago de Chile & Sí & No \\
\hline \multirow{4}{*}{ Colombia (42) } & Universidad de los Andes & No & Sí \\
\hline & Universidad Nacional de Colombia & No & Sí \\
\hline & Pontificia Universidad Javeriana & Sí & No \\
\hline & Universidad de Antioquia & No & Sí \\
\hline Costa Rica (4) & Universidad de Costa Rica & No & Sí \\
\hline Cuba (5) & $\ldots$ & - & - \\
\hline Rep. Dominicana (3) & Universidad Autónoma de Santo Domingo & No & Sí \\
\hline Ecuador (9) & Pontificia Universidad Católica del Ecuador & Sí & No \\
\hline El Salvador (2) & Universidad de El Salvador & Sí & No \\
\hline Guatemala (3) & Universidad Francisco Marroquín & Sí & No \\
\hline Honduras (1) & Universidad Nacional Autónoma de Honduras & No & Sí \\
\hline \multirow{5}{*}{ México (50) } & Universidad Nacional Autónoma de México & Sí & No \\
\hline & Instituto Tecnológico de Monterrey & Sí & No \\
\hline & Universidad Iberoamericana & Sí & No \\
\hline & Instituto Tecnológico Autónomo de México & Sí & No \\
\hline & Universidad Autónoma Metropolitana & No & Sí \\
\hline Panamá (5) & Universidad de Panamá & No & Sí \\
\hline Paraguay (3) & Universidad Nacional de Asunción & No & Sí \\
\hline \multirow{2}{*}{ Perú (17) } & Pontificia Universidad Católica del Perú & No & Sí \\
\hline & Universidad Nacional Mayor de San Marcos & No & Sí \\
\hline Puerto Rico (2) & $\cdots$ & - & - \\
\hline Uruguay (4) & Universidad ORT Uruguay & Sí & No \\
\hline Venezuela (8) & Universidad Central de Venezuela & Sí & No \\
\hline TOTAL & & 21 & 15 \\
\hline$\%$ & & 58,33 & 41,67 \\
\hline
\end{tabular}

Fuente: Elaboración propia a partir de los datos de los portales web de la QS y de cada universidad. 
Las ciencias sociales son ciencias idiográficas enfocadas en la comprensión y explicación de acontecimientos únicos. Estas no difieren para su denominación de las ciencias nomotéticas (como las físicas y naturales dedicadas al estudio de fenómenos reproducibles en condiciones controladas u observables en la naturaleza de acuerdo con un conjunto de condiciones previsibles). Mientras que las primeras tienen por tarea la explicación comprensiva y reflexiva, las segundas se dedican a la formulación de leyes (Tucker, 2012: 276 y ss.). Para establecerse como disciplina en uno $u$ otro tipo de ciencia, un campo de estudio debe contar con un objeto de estudio exclusivo (Cf. Kuper y Kuper, 1996). El primer problema que ello implica en las ciencias sociales es que al estudiar las interacciones humanas el riesgo de solapamiento de los objetos de estudio está siempre presente. Ello se debe a que de una única ciencia de lo social -que comienza su evolución formal en el siglo XIX- se derivaron las disciplinas hoy establecidas (Cf. Porter y Ross, 2003). En el caso de la CP el primer problema con el que nos encontramos es la diferencia entre el plural y el singular. En el mundo latino el plural "ciencias políticas" ha sido dominante, sin embargo, no ha logrado monopolizar la denominación disciplinaria. Las ciencias políticas hoy no difieren de la CP más que por su proyección hacia el pasado que procura ir al rescate de una tradición que llega al Renacimiento. La "ciencia política", por su parte, responde a la búsqueda de la singularidad de la disciplina, un esfuerzo de emancipación que se da en la encrucijada entre los siglos XIX y XX sobre todo en el mundo anglosajón. Pero hoy, en la práctica, las diferencias son imperceptibles, ya que el poder político es el objeto de estudio invariable en unas y otras (Haluani, 1991: 19-20; Caminal, 1996: 24; Almond, 2002: 23-25).

Otro aspecto a ser considerado es el de las subdisciplinas mixtas o fronterizas como la economía política internacional, la geopolítica o la historia diplomática, por ejemplo. En el caso de la primera, la intersección entre la economía y la CP lleva a sus investigadores a enfocarse en la distribución de la riqueza y sus efectos en el poder político (Cf. Gilpin, 1987). Caso parecido es el de la geopolítica, dedicada al estudio de la interacción entre espacio y poder con especial énfasis en las relaciones interestatales (Cf. Spykman, 2008; Walton, 2007). Nuestro tercer ejemplo es la historia diplomática (también entendida en un sentido más amplio como "historia de las relaciones internacionales") (Cf. Renouvin y Duroselle, 2000; Kissinger, 2001). Este ejemplo de subdisciplina fronteriza ha logrado establecerse tanto en los centros de enseñanza de CP como en las de historia, además de aquellas de EE.II., de manera funcional, sin generar conflictos de pertenencia y desarrollando una comunidad multidisciplinaria de investigadores.

Hemos dado tres ejemplos para reducir la larga lista que, en el caso de la CP, podría extenderse hacia la polemología (Cf. Bouthoul, 1984; Howard, 1983) o la psicología política (Cf. Moscovici y Markova, 2006). Campos como estos se presentan hoy como mucho más que programas mutidisciplinarios de investigación, llegando a justificar programas de estudios de pre y postgrado. Pero solo excepcionalmente constituyen escuelas y departamentos universitarios. No así como en los EE.II. Estos últimos se han abierto paso desde temprano en los centros de enseñanza en todos los niveles de la educación superior, pero han tendido a diversificarse, incluyendo a la cooperación internacional, la gobernanza global y las interacciones postestatales. Los internacionalistas se han 
dividido vehementemente de acuerdo con las corrientes teóricas. Este carácter divisivo se ha orientado a la proliferación de subobjetos de estudio, así como a aproximaciones teóricas y metodológicas (Cf. Walt, 1998; Kurki, 2006), diluyendo -formalmente hablandola posibilidad del establecimiento de una disciplina autónoma dentro de las ciencias sociales. No obstante, como hemos visto, la realidad ha apuntado en otro sentido.

Dos escuelas de los EE.II. han sido particularmente claras en distinguir dicha disciplina como autónoma de la CP: la británica y la española. La escuela británica se desarrolló de forma autónoma tras el final de la Primera Guerra Mundial, específicamente con el establecimiento de la primera cátedra de asuntos internacionales en la Universidad de Gales: la Cátedra Woodrow Wilson. En retrospectiva resulta paradójico que su primer titular haya sido el realista E.H. Carr. La escuela española es más reciente, pero también más vehemente en la distinción de los EE.II. como disciplina emancipada de la CP. El desarrollo posterior de la escuela inglesa da cuenta de esta emancipación aunque no desde la CP, sino desde la sociología. Los trazos de esta escuela se encuentran fundamentalmente en la obra de Headley Bull (2002), quien abrió el camino para la vía media que la escuela inglesa ha querido sostener entre el realismo hobbessiano y el idealismo kantiano proponiendo el concepto de "sociedad internacional", la que reclama como heredera de una tradición grociana. Esta tendencia europea también se halla en la obra de Antonio Truyol y Serra (2006). Con un origen más cercano al derecho, y con una mayor proximidad a la tradición kantiana, la escuela española, al igual que la inglesa, se compromete con el concepto de sociedad internacional y disciplinariamente afirma la autonomía de los EE.II. A partir de este ha florecido una escuela cuyo mayor énfasis es distinguir la ciencia de lo internacional de la estadounidense y dominante escuela politológica (Cf. Hoffmann, 2001; Del Arenal, 1990).

A pesar de las tensiones internas, y de las dificultades al momento de encontrar un objeto de estudio distintivo y metodologías de investigación claras, los EE.II. han proliferado y adquirido espacios propios. Así lo demuestra el crecimiento de la International Studies Association, una asociación más joven, pero mucho más cosmopolita y específica que la American Political Science Association y la International Political Science Association. El creciente número de escuelas y departamentos dedicados a los EE.II. fuera del ámbito de la CP también son muestra de esa autonomía en la práctica. Incluso en departamentos multidisciplinarios la CP y los EE.II. han tendido a separarse, al menos operativamente.

En suma, tenemos que los EE.II. han venido reclamando con cada vez mayor éxito su propio espacio entre las ciencias sociales. Su éxito como ciencia social estadounidense -como los calificara Hoffmann (2001)- ha venido acompañado de un éxito progresivo en el resto del mundo. Sus avances institucionales demuestran que, en efecto, hay una distinción práctica entre estos y la $\mathrm{CP}$, no obstante, sus inherentes debilidades en la definición de un objeto de estudio inequívoco (¿paz y guerra, cooperación/conflicto económico, diplomacia, globalización o estudios regionales?), su pluralidad "paradigmática" (realismo, idealismo, racionalismo y constructivismo, y su falta de métodos distintivos). Si bien -como expone Hellmann (2011) - el avance metodológico de los EE.II. ha sido impresionante, este se ha sostenido sobre una plataforma de préstamos primero desde 
las humanidades y después desde las ciencias sociales, especialmente desde la economía y la CP (2011: 1312-1314). Aunque incompleta y con resultados dispares en el mundo académico, no se puede negar que la realidad arroja una tensa emancipación parcial de los EE.II., lo que le ha permitido desarrollar un rico mundo interior de debates teóricos. Sin embargo, y como veremos a continuación, un potencial ascenso de la corriente teórica del realismo neoclásico podría poseer la fuerza eventual para, si no revertir esa realidad, al menos, ofrecer mayor resistencia a la misma.

\section{REALISMO NEOCLÁSICO}

El auge del realismo neoclásico crea una cortina de humo que amenaza con negarnos la posibilidad de comprender las implicaciones de la teoría detrás del fenómeno académico. Debemos evitar caer en la falsa creencia de una evolución paradigmática del realismo, así como del resto de las teorías rivales en los EE.II. El caso del realismo neoclásico puede ser enmarcado como un modelo de desarrollo teórico dialéctico antes que kuhniano ( $C f$. Pérez Toribio, 2011), pues su desarrollo parte de la restauración de preceptos del realismo clásico, pero en el marco referencial estructuralista del neorrealismo (Cf. Rose, 1998). Con esto nos referimos a que el realismo neoclásico sigue atendiendo, en primer lugar, a la distribución de capacidades en el sistema internacional como su variable independiente, incorporando además factores de política doméstica como variable interviniente.

El realismo neoclásico es una teoría de la política exterior. Esto parece obvio al referirnos a cualquier teoría de las relaciones internacionales, pues como los realistas clásicos aspiraron, el realismo debe ser una guía de acción en el marco de la política internacional (Cf. Morgenthau, 1986; Carr, 2004; Niebuhr, 1953). En un sentido contrario, fue el realismo estructural o neorrealismo, distanciándose del primero a partir de la rigurosidad de los métodos que incluyó, en lo concerniente a los conceptos de anarquía y estructura internacionales, ya que su objetivo era menos ambicioso y menos interesado a servir a los estadistas, al menos en primer lugar (Cf. Waltz, 1979; May et al., 2010). El neorrealismo se preocupó por entender la lógica de la dinámica política internacional y no a la política exterior de cada Estado (Cf. Waltz, 1996). Adicionalmente, el neorrealismo, preocupado principalmente por la estructura internacional, abandonó las consideraciones éticas implícitas en el realismo clásico dando primacía a la descripción fenomenológica antes que a la prescripción normativa (Cf. Lebow, 2003).

La etiqueta realista neoclásica fue colocada sobre aquellos trabajos que, dándole importancia a la estructura internacional en tanto particular distribución de poder en el sistema, consideran a la política exterior como variable dependiente y a los complejos procesos de política interna (capacidad y percepción de las élites) como variables intervinientes. El autor que puso esta etiqueta a partir de un conjunto de artículos y libros seleccionados trató de rastrear los antecedentes de este enfoque en auge dentro de la moderna tradición anglosajona del campo de la historia de las relaciones internacionales (Rose, 1998). Tres obras son especialmente señaladas como pioneras en la distribución internacional del poder como explicación: las de Robert Gilpin (1987), Paul Kennedy 
(1987) y Michael Mandelbaum (1988). En materia de percepciones sobre esos cambios en la distribución internacional de las capacidades, se destaca la labor de Robert Jervis (1976), una incorporación de enfoques de la psicología aplicados para explicar cómo, por qué y cuándo los líderes perciben y actúan ante una configuración de poder dada.

Pero el realismo neoclásico no es necesariamente novedoso en el sentido expuesto, aunque resulte perceptible su reciente auge académico. Más recientemente (y aunque pertenezca a lo que ya es una tradición de pensamiento politológico, la "elección racional") Charles Glaser ha expuesto la utilidad del racionalismo en la política internacional. De acuerdo al autor, la variables materiales -aquellas que estarían más asociadas al neorrealismo en su búsqueda de condiciones definitorias de la estructura internacional y orden generadodeben estar acompañadas de variables de información que permitirían identificar la capacidad de un Estado (asumido por Glaser como un actor racional) para procesar los datos provenientes del sistema internacional y actuar en consecuencia (2010: 3-6; 85-87). La tesis de Glaser no contraviene al realismo en su forma clásica como sí cuestiona el efecto "bolas de billar" o de "caja negra" del Estado, en tanto se preocupa por el estudio de la decisión en política exterior, particularmente en el desarrollo histórico de la misma sobre la base del procesamiento de la información por parte de los responsables políticos, en especial en temas sensibles de seguridad y defensa.

Como acertadamente señala Dario Battistella (2012) en el caso de Raymond Aron, podríamos encontrar en retrospectiva ejemplos de antecedentes del realismo neoclásico, o en palabras de Battistella, "realismo neoclásico vanguardista" (2012: 371). Influido por la École des Annales, Aron (1963) plantea un análisis que combina el estudio de la distribución de las capacidades potenciales en el sistema internacional, pero a su vez considera los factores biográficos, psicológicos e ideológicos que rodean a cada estadista. Aron se negó al análisis puramente histórico al estilo de la metodología del realismo clásico anglosajón, pero también al análisis estructural-racionalista tal y como luego lo plantearía el neorrealismo. Una aproximación realista dinámica es la propuesta aroniana, tan apegada a la realidad material como a la igualmente cruda realidad del peso de los miedos y las motivaciones íntimas de los decisores (Battistella, 2012: 380 y ss.).

Pero así como Rose y los demás realistas neoclásicos ignoran a Aron, Battistella omite el método planteado por otros dos franceses: Pierre Renouvin y Jean-Baptiste Duroselle (2000). Ambos autores ensayaron modelos de análisis y explicación histórica internacional destacándose, en el caso de Renouvin, el concepto de "fuerzas profundas" o condiciones objetivas bajo, sobre y con las cuales debe trabajar el estadista (1990). Duroselle, por su parte, avanzó en su propuesta de teoría de las relaciones internacionales en un trabajo que más podría ser calificado de teoría de la política exterior. Este autor penetra en el carácter del estadista, pero además señala un aspecto que hoy es considerado característicamente realista neoclásico: que la unidad política básica no es el individuo ni el Estado, sino el grupo decisor (Duroselle, 1998). El realismo neoclásico ha adoptado el término de EPE para destacar a este grupo de políticos, burócratas y lobistas, civiles y militares, que rodean al decisor final, que condicionan sus preferencias y que además juegan un rol decisivo en la ejecución (Lobell, 2009: 43 y ss.). 
En América Latina, si bien el desarrollo intelectual se ha inclinado hacia la corriente de la teoría de la dependencia (Cf. Cardoso y Faleto, 1979), el trabajo teórico de Escudé bien podría ser incluido en la lista de tempranas aproximaciones a lo que luego se llamó realismo neoclásico. De acuerdo con este autor: "Para un país periférico, vulnerable, empobrecido y poco estratégico para los intereses vitales de las potencias centrales, la única política exterior moral es aquella que reduce los costos y riesgos de costos eventuales, maximiza beneficios y, por sobre todo, atrae inversiones y baja las tasas de riesgo-país" (1992: 281-282). Escudé presenta una teoría para Estados débiles o poco poderosos que no producen, sino que aceptan normas. El "realismo periférico" sigue la lógica de la teoría de la dependencia diseccionando al mundo en centro y periferia (o Norte-Sur), pero además agrega a su estructuralismo implícito el problema de la agencia (Cf. Wendt, 1987) incluso en términos de moralidad, refiriéndose al proceso de toma de decisiones y a la responsabilidad del estadista. Aquí la unidad de la naturaleza de lo político queda expuesta.

Estos antecedentes y supuestos sugieren que en el realismo neoclásico hay un nuevo intento de retorno a la unidad de lo político. Este se refiere a la existencia de patrones comunes (o al menos articulados por el proceso de política exterior) entre la política interna y la política internacional, lo que contradice el argumento según el cual lo internacional responde a condiciones únicas de interacción que escapan de la lógica política interna, en tanto una sociedad internacional no tendría como centro al Estado (siendo el último el centro natural de los sistemas políticos nacionales y de allí el desarrollo de la teoría política), sino a la interacción social internacional que necesariamente incluye a otros actores (Del Arenal, 1981: 854-857). Esto constituye la base del criterio de autonomía disciplinaria. No obstante, para Kenneth Waltz, pasar de un sistema a otro en el momento en el que se asume como principio ordenador la jerarquía en lugar de la anarquía, o viceversa, no implica en ningún momento la separación ontológica de lo político como compleja realidad condicionada por el poder, la voluntad, el miedo y los valores morales (1979: 79 y ss.; 1954: 16 y ss.). La unidad de lo político rescata los fundamentos filosóficos de los pensadores que son considerados ancestros del realismo, desde los soldados Tucídides y Clausewitz, pasando por los filósofos Maquiavelo y Hobbes.

La proliferación de enfoques y teorías sobre las relaciones internacionales da cuenta de la vitalidad del debate, brindando una vibrante diversidad que permite refinar conceptos, modelos y teorías al calor de la polémica. No obstante, un efecto colateral de esa diversidad es que la proliferación atenta contra la posibilidad de constituir lenguajes académicos comunes para este campo de la CP. El realismo neoclásico puede ser percibido de dos modos opuestos. Por una parte, como una nueva reinterpretación del realismo o, para ser más exactos, del neorrealismo. Bajo esta mirada se expone el problema de la proliferación teórica o, en este caso, subteórica, que apunta a la dispersión y a la pérdida de criterios comunes en las relaciones internacionales (Cf. Brooks, 1997; Snyder, 2002). Por la otra, puede ser visto como el producto sintético del neorrealismo, como teoría sistémica estructural, y el realismo clásico, como explicación de la conducta de los Estados. Esta segunda propuesta parece más acertada, porque además incorpora elementos cognitivos que le permiten al realismo avanzar hacia la aspiración de ser una teoría multinivel 
(sistémico-internacional, subsistémico-regional y estatal) capaz de ofrecer explicaciones sobre la política internacional sin dejar de lado las causas regionales y domésticas de la política exterior (Cf. Jervis, 1976; Putnam, 1988; Allison y Zelikow, 1999).

Una parte importante del debate realista hoy, cuando la versión neoclásica luce en auge, es cuál es el lugar que ocupan los valores éticos en el ejercicio del poder. El éxito de las tesis waltzianas trajo como consecuencia el abandono de factores normativos que habían acompañado al realismo como tradición de pensamiento, incluso desde antes de ser bautizado como realismo. Los trabajos de Michael Williams (2005) y Richard Lebow (2003) desafían esa visión dominante de amoralidad instrumental del neorrealismo, retomando, entre otros conceptos, uno de especial aprecio realista: el interés nacional. A partir de una preocupación por la política exterior, esta crítica apunta a un redescubrimiento de las motivaciones exteriores de los decisores políticos basándose en consideraciones no puramente racionales, así como a la ruptura en la compartimentación entre las ciencias sociales y las humanidades. La relación de esta crítica con el realismo neoclásico no se expone expresamente por ninguno de los dos autores. No obstante, una y otra expresan la preocupación por la vuelta a la reflexión sobre los objetivos políticos definidos por agentes con miedos, intereses, ideologías y compromisos. Se unen ambos a la preocupación del constructivismo por el rol del agente en la estructura (Cf. Wendt, 1987).

De acuerdo con uno de sus principales exponentes: "Neoclassical realism suggests that state power -the relative ability of the state to extract or mobilize resources from domestic society as determined by the institutions of the state, as well as by nationalism and ideology- shapes the types of internal balancing strategies a state is likely to pursue" (Taliaferro, 2006: 467). ${ }^{4}$ El realismo neoclásico comparte con el neorrealismo el carácter estructuralista así como la mayor parte de sus supuestos generales, aunque con variaciones que lo refinan (Cf. Schweller, 1997). Anarquía internacional, distribución de capacidades como variable principal a considerar, y procura de seguridad -aunque entendida como control y/o transformación del entorno inmediato (Taliaferro, 2006)-, siguen siendo elementos centrales para los neoclásicos (Rose, 1998: 146-147). El sistema internacional consta de un orden plural y descentralizado que somete a sus unidades a una constante interacción directa. El factor ordenador anárquico persiste sin cambios desde el neorrealismo en el pensamiento neoclásico. No niega este último el valor de las instituciones multilaterales como sí lo hace la variante ofensiva del primero ( $C f$. Mearsheimer, 1994), pero sí rinde tributo al rol del Estado como institución y actor central de la política internacional. Citando nuevamente a Taliaferro: "Neoclassical realism incorporates the complex model of state-society relations implicit in classical realism, while building upon neorealism's insights about the constrains of anarchy and the relative distribution of material power" (2006: 470). ${ }^{5}$

4 Tr. El realismo neoclásico sugiere que el poder estatal -la capacidad relativa del Estado para extraer o movilizar recursos de su sociedad que está determinado por las instituciones, así como por el nacionalismo y la ideología- da forma a los tipos probables de estrategias de balanceo interno que un Estado podría seguir. Tr. El realismo neoclásico incorpora el complejo modelo de relaciones entre Estado y sociedad implícito en el realismo clásico, junto con las ideas del neorrealismo acerca de las limitaciones de la anarquía y la distribución relativa del poder material. 
En un orden anárquico, el principio de la autoayuda sigue siendo un imperativo de ese actor central que es el Estado. Pero un importante aporte es el de ir más allá de la visión realista aceptada con relación al poder y las amenazas. En el realismo neoclásico la posición que un Estado ocupa con respecto a la jerarquía de capacidades del sistema que le es relevante es una variable de análisis fundamental, pues las capacidades no son un fin en sí mismo, ni todos los intereses pueden ser satisfechos a través de estas, ni es posible contar con una herramienta de análisis que solo considere la hegemonía como objetivo. Al intentar mejorar su posición de poder mediante capacidades, un Estado está reaccionando a: (i) las condiciones que le ofrece el sistema internacional, (ii) las amenazas tal y como resulta efectivamente capaz percibirlas, pues la evaluación racional está condicionada por el acceso a la información y los valores de los individuos responsables de la toma de decisiones ejecutivas en materia de política exterior y de defensa, (iii) la capacidad real que tienen esos decisores o estadistas para aprovechar, y hacer ver a sus contrapartes extranjeros, las capacidades reales o supuestas que pueden extraer de su Estado en función de la acción exterior, y de forma general y última (iv) tratando de hacerse con mecanismos propios para mejorar sus oportunidades de controlar su entorno inmediato.

Si consideramos en su conjunto los cuatro objetivos expuestos, es posible concluir que según el realismo neoclásico el Estado, más que seguridad o poder, está en la procura de instrumentos que le permitan analizar exitosamente la incertidumbre (Lobell, 2009: 46-54; Rathbun, 2007: 538-541), sobre todo si nos colocamos en un ambiente internacional multipolar. Consideramos que esta afirmación es correcta y que acierta en la redefinición del realismo como una explicación acerca de lo que los actores internacionales realmente quieren, pero al mismo tiempo tomamos en cuenta que no se puede entender la política exterior de los Estados sin estimar que las capacidades materiales condicionan la extensión de los objetivos del actor, o en palabras de Rose: "The central empirical prediction of neoclassical realism is (...) that over the longterm the relative amount of material power resources countries possess will shape the magnitude and ambition (...) of their foreign policies..." (Rose, 1998: 152). ${ }^{6}$

Los gobiernos que perciben las oportunidades de tomar decisiones con mayor libertad y tienen un mejor acceso a sus propios recursos ponen a la autonomía como un objetivo indispensable para fines tantos nacionales como exteriores. Así, el estudio del poder en el realismo neoclásico se expande más allá del poder nacional, es decir, del conjunto de capacidades que posee un Estado, sino que se adentra a lo que Fareed Zakaria denominó "poder estatal", es decir, esa parte de las capacidades nacionales potenciales que el gobierno puede extraer en función de sus propósitos políticos (1998: 9). Así, yace en el realismo neoclásico la necesidad de ir hacia una teoría que también explique las variables al nivel de las unidades del sistema internacional a nivel del Estado, en especial a nivel de capacidades gubernamentales (Cf. Wohlforth, 1993; Snyder, 1993; Zakaria, 1998; Schweller, 1998).

6 Tr. El realismo neoclásico incorpora el complejo modelo de relaciones entre Estado y sociedad implícito en el realismo clásico, junto con las ideas del neorrealismo acerca de las limitaciones de la anarquía y la distribución relativa del poder material. 
Es posible ver la traza del neorrealismo en el realismo neoclásico, pero también su voluntad por rescatar lo mejor del realismo clásico. Cuando Morgenthau hablaba de poder ver por encima del hombro del estadista y aproximarse a la comprensión de sus acciones exteriores, estaba sugiriendo una teoría de la política exterior junto con la propuesta de una teoría de las relaciones internacionales. En el realismo clásico no parecía haber ningún problema en distinguir una cosa de la otra. Como teoría de las relaciones internacionales, el realismo sería tan capaz de explicar al sistema internacional, como a las acciones de los Estados en éste. La discusión no cabía en tanto se entendía que debía haber una política exterior racional, a saber, una política exterior realista. Este rasgo prescriptivo que se presenta en Morgenthau de forma análoga a como se presentó en Clausewitz con el fenómeno de la guerra limitada, asechó al neorrealismo hasta las aclaratorias de Waltz (1996: 54-7). Con el auge waltziano, el neorrealismo queda como una explicación de la política internacional que no es capaz de adentrarse en las acciones de los estadistas. A diferencia de ello, el realismo neoclásico sí hace distinción entre política internacional y política exterior como variables de estudio. Y esta corriente es, sobre todo, una teoría de la política exterior. No abandona las explicaciones estructuralistas, sino que incorpora elementos del realismo clásico y de las incursiones de la psicología en el estudio de la realidad internacional. Con el realismo neoclásico volvemos a asomarnos sobre el hombro del estadista. Factores domésticos como el consenso entre las élites, la vulnerabilidad de los gobiernos y regímenes, así como la cohesión social y de élites (Schweller, 2004: 168-181) refuerzan la necesaria atención de los EE.II. en los asuntos políticos internos de cada sistema nacional.

Resumiendo los presupuestos realistas neoclásicos podemos decir que, en primer lugar, la teoría es una refinación de las escuelas realistas clásica y neorrealista, un esfuerzo por combinar lo mejor de ambas en vista de una explicación integral de la política internacional. El resultado es una teoría realista multinivel. Segundo, la distribución de capacidades, en especial de capacidades duras (demográficas, económicas y militares) y constituye la variable independiente para la comprensión parcial de la política exterior, de allí la importancia del estudio de los órdenes de acuerdo con la polaridad y concentración internacional. Tercero, el realismo neoclásico reconoce la influencia de la política doméstica, así como de los factores psicológicos del estadista y las corrientes ideológicas dominantes de cada régimen político. Además, incluye factores que podríamos considerar de gobernabilidad y que van desde la cohesión social hasta la cohesión de las élites, pasando por el consenso dentro y la vulnerabilidad relativa de los órdenes internos. $Y$ cuarto, la teoría representa el mejor esfuerzo por construir un puente entre el estudio de la política internacional y la comprensión de los factores domésticos que, junto a los sistémicos, determinan a la política exterior (Rose, 1998: 153; Cf. Taliaferro et al., 2009).

\section{UNIDAD DISCIPLINARIA: EL OBJETO DE ESTUDIO}

Como se señaló antes, la autonomía que presentan los EE.II. es evidente en términos prácticos. Más desde el punto de vista disciplinario, esto es, desde la perspectiva de 
una ciencia social no parece haber razones suficientes para separarlos de la CP. Para Colin Wight, la filosofía de las ciencias sociales debe abordarse a partir de dos pares de categorías dicotómicas: estructura/agente y explicación/comprensión (2002: 24). La relación entre estructura y agente es central en el debate teórico de los EE.II. (Cf. Wendt, 1987), y en el realismo neoclásico se manifiesta de manera convergente en la interacción entre las variables independiente e interviniente.

En el caso del realismo neoclásico, la causalidad sigue siendo la principal preocupación y no así la comprensión interpretativa. La preocupación acerca de los factores de política doméstica que afectan el desempeño del ejecutivo de política exterior no pasan por el interpretativismo (aunque en un sentido weberiano siempre se deba considerar la interpretación como un paso científico previo al establecimiento de la causalidad), sino por la explicación estructural en el sistema político interno. El realismo neoclásico, en consecuencia, parte de un análisis estructural al igual que el neorrealismo, pero en dos niveles (o tres, si se considera el nivel regional), siendo el EPE la unidad de análisis integradora de los niveles.

El realismo neoclásico no solo es realista en el sentido teórico, sino que también lo es en el sentido ontológico. Como teoría del ser, la ontología se pregunta por la realidad del mundo. Sus posiciones se resumen en esencialista y fundacionalista. La primera posición asume que la realidad se construye a partir de percepciones, mientras que la segunda parte de la existencia de una única e independiente realidad (Marsh y Furlong, 2010: 18). El realismo ontológico es fundacionalista al igual que el positivismo, pero a diferencia de este último, se fía menos de la observación directa aceptando que hay relaciones estructurales a las que no se puede acceder y que solo son verificables a través de manifestaciones posteriores (Ídem: 20). El realismo ontológico es palmario en el caso del realismo neoclásico, sobre todo en el tratamiento de los aspectos más espinosos de la variable interviniente. En los casos de teorías distintas, la ontología interpretativista y la misma positivista también han encontrado espacios en los EE.II. y la CP. Lo mismo podría decirse del resto de las ciencias sociales, por lo que el criterio ontológico, aunque de vital importancia, no nos ayuda a dilucidar el problema de la unidad disciplinaria.

En la epistemología podríamos intentar hallar claves sobre la relación autónoma o unitaria de los EE.II y la CP. Como teoría del conocimiento, esta trata de la forma en la que el sujeto aborda al objeto para aprehenderlo. En la $\mathrm{CP}$, así como en los EE.II., la distinción generalmente aceptada es la de epistemología positivista, comprometida con la explicación, y epistemología hermenéutica, orientada hacia la comprensión interpretativista (Ídem: 19-20). El realismo neoclásico responde epistemológicamente a la posición positivista, en tanto busca establecer una cadena de hechos causales que respondan a las variables que explican una(s) determinada(s) conducta(s) exterior(es). Pero como la ontología, la epistemología nos puede ayudar a distinguir entre los rasgos de una teoría y otra, pero no entre una y otra disciplina social. Por tanto, cualquier cosa que se diga desde las posiciones epistemológicas no resulta definitivo en lo referente a la unidad o autonomía disciplinaria. 
El rasgo estructuralista del realismo neoclásico abre las puertas a la metodología desarrollada en la $\mathrm{CP}$, sobre todo en el plano de la racionalidad en el marco de procesos de decisión y acción colectiva (Cf. Ostrom, 1998). Metodológicamente se gestan grandes espacios de convergencia entre los EE.II. y la CP, porque, primero, cuentan con la posibilidad de sofisticar sus análisis y llenar sus vacíos analíticos con instrumentos para hacer operacionalizables las variables de estudio, mientras que la CP incubada en el racionalismo de la escuela anglosajona (en particular la estadounidense), rompería con el parroquialismo de los estudios institucionales, pudiendo proyectar sus resultados más allá del ámbito de la política comparada hacia un ámbito verdaderamente internacional. El realismo neoclásico, por su ontología realista, contempla métodos cuantitativos para aquellas variables observables y cualitativos para aquellas que no lo son. Pero la metodología tampoco nos ofrece una respuesta definitiva, ya que sigue sin diferenciar entre una ciencia y otra, solo entre teorías.

Hemos expuesto sucintamente los aspectos ontológicos, epistemológicos y metodológicos que podrían compartir la CP y los EE.II., siempre con atención al realismo neoclásico, con el fin de descartar respuestas alternativas a lo que consideramos como el indivisible punto de conexión entre la una y los otros, el objeto de estudio: el Estado como entidad de poder (Caminal, 1996: 21-23; Haluani, 1991: 76-77). Los estudios politológicos e internacionales no escapan de esa realidad. Resulta inadmisible la existencia de dos ciencias con un mismo objeto de estudio, siendo que las disciplinas científicas se definen por su objeto. El realismo neoclásico, como forma más novedosa de la larga tradición de pensamiento político realista, tiene la virtud de reincorporar al Estado en el debate. No es casual que esta versión del realismo coincida y florezca en medio de un proceso de multipolarización del sistema internacional que, por reducir la rigidez de los compromisos respecto a liderazgos hegemónicos y mecanismos multilaterales, tiende a ofrecer amplias oportunidades de autonomía externa y poder interno a los Estados. La vuelta del Estado al centro del debate politológico e internacional, junto con el auge del realismo neoclásico, pone de relieve la mayor coincidencia entre los EE.II. y la CP.

\section{V. ¿RETORNO A LA CIENCIA POLÍTICA?}

De acuerdo con lo expuesto, el realismo neoclásico posee elementos que podrían ser considerados la base de un sólido cuestionamiento contra la idea de emancipación de los EE.II. respecto a la CP. La inclusión de elementos de política interna como variable interviniente en la ejecución de la política exterior, y los efectos de esta última en la política internacional en general, debilita la tesis de los EE.II. como una esfera del conocimiento autónoma dentro de las ciencias sociales. La posibilidad de que el realismo neoclásico genere un efecto de retorno de los EE.II. a la $\mathrm{CP}$, o que al menos debilite los argumentos autonomistas, dependerá en gran medida de la difusión real de la teoría. Asumimos que la misma se encuentra en auge y que ello se puede constatar en el creciente interés que ha generado en los últimos quince años como puede verse en el Gráfico 1: 
Gráfico 1. Crecimiento de publicaciones sobre Realismo Neoclásico

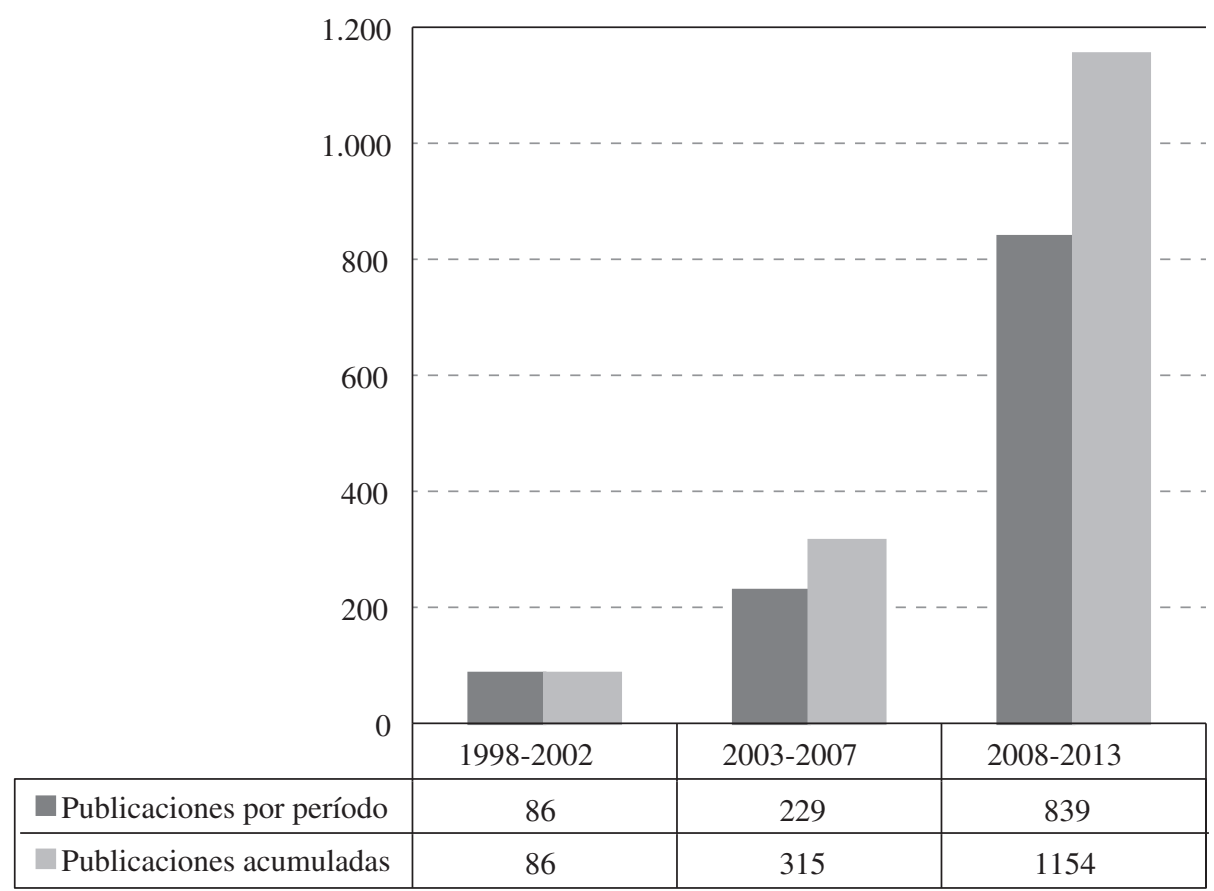

Fuente: Google Scholar.

Una búsqueda por períodos del término "'neoclassical realism' + 'international relations'" realizada en el motor de búsqueda académica Google Scholar el 19 de diciembre de 2013, arrojó como resultado un crecimiento de un 266\% entre el período 1998-2002 y el 20032007 , y de un $366 \%$ entre el último período y el 2008-2013. Tomamos como referencia el término en inglés por la amplia difusión e impacto de este idioma en la CP en general y en los EE.II. en particular, además de tomar en cuenta el hecho de que en las publicaciones arbitradas en otros idiomas es usual que se incluyan resúmenes y palabras clave en inglés. 1998, el año de inicio de la búsqueda, responde al año de publicación del ya citado trabajo de Gideon Rose. No se incluyen citas de trabajos anteriores para evitar incrementar equívocamente el número de entradas encontradas. Se incluyó el término "international relations" para evitar cualquier tipo de confusión con las corrientes neoclasicistas y realistas en el arte.

Vistos los datos, surgen tres interrogantes respecto a la posibilidad de una reunificación de los EE.II. a la CP: ¿es necesaria, deseable y/o posible?

Definir necesidades en política es difícil porque median intereses que no son siempre públicos. No es muy distinto en el mundo académico. Sin embargo -y de conformidad con la motivación de este ensayo- no son los problemas administrativos sino los académicos los que definen la necesidad (así como la deseabilidad y posibilidad). 
La necesidad es una condición negativa, misma que sugiere carencia. La CP hace un amplio uso de técnicas y métodos provenientes de ciencias auxiliares. Pasando por la sociología, la historia, la geografía, la antropología y la economía, la CP podría tener serios problemas de identidad particular de no ser porque posee un objeto de estudio claramente diferenciado: el Estado en tanto manifestación central del poder. La emancipación de los EE.II. respecto a la CP los deja en una situación de indefensión como disciplina científica. Lo internacional no goza de cualidades para ser un objeto de estudio científico social independiente. El sistema internacional es político y su principal cualidad es poseer una estructura anárquica; según Waltz, cuya teoría (el neorrealismo) es pilar autonomista de los EE.II. (1979). Siguiendo con Waltz, se pasa de un sistema a otro cuando el principio ordenador cambia. Así, los órdenes políticos internos (sistemas políticos nacionales) estarían idealmente regidos por el principio jerárquico, mientras que el orden político internacional (sistema internacional) lo estaría por la anarquía (1979: 88 y ss.). El paso de un principio a otro es el paso de un sistema a otro, pero no sugiere Waltz un cambio ontológico de la realidad política, ni un cambio epistemológico en su abordaje científico, ni mucho menos el cambio del objeto de estudio, que para el autor es, de forma destacada, el poder, cuya más perfecta manifestación es el Estado. El retorno de los EE.II. a la CP sería necesario para evitar que los primeros sigan careciendo de un objeto de estudio aprehensible.

Lo deseable de este retorno no debe buscarse en motivaciones personales o administrativas, sino en consideraciones científicas. No es solo la necesidad de los EE.II. lo que haría deseable el retorno pleno y reconocido, sino el fortalecimiento de la CP como disciplina. La dispersión analítica que supone sostener la artificial e insatisfactoria distinción entre órdenes políticos interno e internacional, crea a su vez una dispersión de esfuerzos y recursos materiales e intelectuales. El realismo neoclásico, sin proponérselo, apunta en esta dirección, concentrándose en el punto de contacto entre ambos órdenes políticos: la política exterior. Asumir a la política exterior como variable dependiente no es olvidar al resto de las variables sino, por el contrario, apelar a una comprensión más profunda y exacta sobre la distribución de poder en el sistema internacional, las condiciones para la gobernanza y la cooperación, así como sobre la naturaleza de los regímenes políticos internos, los sistemas de partidos / facciones, las ideologías, la relación Estado-sociedad, las relaciones cívico-militares, los conflictos de intereses intranacionales y la psicología de los líderes. Complementar las explicaciones acerca de la realidad política como un todo reforzaría a la CP y a los EE.II. dentro del amplio rango de las ciencias sociales y de la ciencia en general.

Pero por más que parezca académicamente necesario y deseable, la plausibilidad sigue siendo un problema para la reunificación. El auge del realismo neoclásico no quiere decir de antemano que la teoría logre alcanzar la hegemonía en los EE.II. El liberalismo (Cf. Doyle, 1986; Mansfield y Milner, 1997) y el constructivismo social (Cf. Wendt, 1999; Barnett y Duvall, 2005) siguen siendo fuertes contendientes, por no mencionar las explicaciones realistas defensivas (Cf. Glaser y Kaufmann, 1998) y ofensivas (Cf. Mearsheimer, 2001). Aquí es donde se halla el punto débil en la hipótesis de la indiscutible reunificación disciplinaria. Pero tal aspiración no procedería de ningún modo 
automáticamente, ni mucho menos de forma coordinada, pues el mismo crecimiento en el número de investigadores especializados en los EE.II. y la presión por el desarrollo de refinamientos teóricos cada vez más específicos hacen que sea una "comunidad científica" no uniforme. La escasa plausibilidad descansa entonces en una alternativa a las grandes revoluciones científicas homogenizantes, nos referimos a la debilidad del argumento contrario a la pertenencia de los EE.II. a la CP. Aunque ello no implique la modificación de las estructuras asociativas hoy tendientes a la parcial independencia disciplinaria, sí plantear el potencial reforzamiento de las tendencias a la reunificación indiscutible, y una teoría de creciente dominio en la explicación de la política exterior, bisagra entre el sistema político nacional y el internacional, podría traer consigo este inadvertido efecto secundario.

\section{UNA AGENDA DE INVESTIGACIÓN PARA LA REUNIFICACIÓN}

Aún en el mejor escenario para la proliferación de criterios realistas neoclásicos en los EE.II., una reunificación disciplinaria no está garantizada, ni resulta necesariamente deseable. El desarrollo de una agenda de investigación dentro de un particular marco teórico depende de la conjunción entre necesidad provista por la realidad y voluntad que parte del investigador. De nuevo las tensiones no excluyentes entre estructura y agencia se presentan en los EE.II., aunque esta vez incursionando en el plano disciplinario. La tendencia multipolar, vista en el sentido waltziano de distribución de capacidades en el sistema internacional, resulta en una tentación estructuralista tendiente a fortalecer la autonomía disciplinaria. No obstante, la proliferación de estudios orientados a la comprensión y explicación de la política exterior en sus procesos y resultados ${ }^{7}$ hace que observar más de cerca los procesos decisionales desde el Estado y no solo sus causas y resultados en el sistema se convierta en un asunto ineludible. La evolución actual del orden mundial y de la disciplina parece conducirnos a una conjunción entre necesidad y voluntad, lo que reforzaría la agenda de investigación que ya está en marcha.

Desde que Gideon Rose escribió la revisión bibliográfica sobre las cuatro explicaciones que identificó respecto al análisis de política exterior dando nombre al realismo neoclásico (1998), mucho y poco se ha avanzado en la agenda de investigación. Mucho, pues como se mostró en el Gráfico 1, cuantitativamente ha crecido el interés por la integración que hace el realismo neoclásico que, sin ser novedosa, ha logrado un significativo impacto gracias, entre otras cosas, a los cambios en el orden internacional, que han implicado el reconocimiento de un mundo más complejo en el que la comprensión y explicación de

$7 \quad$ Haciendo uso de la herramienta Ngram de Google Books es posible ver un incremento en las obras en inglés publicadas en forma de libro y que contienen la materia "Foreign Policy Analysis", en especial desde 1995 (luego de una caída que coincide con el optimismo anglosajón posterior al fin de la Guerra Fría). En castellano se registra un incremento similar, aunque el término "Análisis de Política Exterior" no esté tan generalizado en este idioma. https://books.google.com/ngrams. Otro indicador de dicho incremento es el lanzamiento de la revista académica Foreign Policy Analysis de la International Studies Association en 2005. Muestra del crecimiento del campo de estudio que mejor integra factores de política doméstica como intervinientes en política internacional. 
la política internacional resulta incompleta sin el concurso de un análisis de la realidad interna de los Estados. Y poco en la medida en que la riqueza de la agenda de investigación no ha acompañado al ritmo cuantitativo que ha despertado su interés. A partir de la revisión de una muestra del 10\% de artículos, tomada del universo de documentos con los que se elaboró el Gráfico 1, se identificó que el realismo neoclásico ha estado principalmente enfocado en la discusión teórica. Esto se le puede adjudicar al sesgo en los términos de la búsqueda, así como a la relativa juventud de la propuesta, que además surge en medio del auge de alternativas teóricas dentro de la corriente reflectivista.

La segunda tendencia en la agenda de investigación que se ha seguido hasta ahora está referida a los estudios históricos. El acceso a documentos diplomáticos de las grandes potencias ha permitido una indagación revisionista del último siglo de historia diplomática. Con un énfasis en la Guerra Fría, que por causa del centenario se ha desplazado a la Gran Guerra, el realismo neoclásico podría ser identificado como una teoría aplicable casi exclusivamente a casos históricos en donde se pueda contar con documentos de libre acceso y cuyo método central sea la investigación en archivos. La tercera tendencia está asociada a la política exterior de las grandes potencias, principalmente los Estados Unidos, la Unión Soviética y los poderes europeos occidentales, con especial atención a las potencias modernas del Reino Unido, Francia y Alemania. Este derrotero ha conducido a la agenda de investigación en una dirección en la que los actuales eventos de transformación del orden mundial no parecen tener cabida, aunque la teoría referida ofrezca destacables capacidades de análisis contemporáneo.

El desarrollo del realismo neoclásico, del que podría depender en buena medida una reunificación disciplinaria, con los EE.II. reintegrándose a la $\mathrm{CP}$, estaría sujeto a la superación de estos estadios primarios representados en las tres tendencias detectadas. En esa dirección, la primera tendencia debe, sin abandonar el debate teórico, orientarse hacia un debate metodológico que incluya a los clásicos modos de entender el estudio de la política exterior como el racional, el organizacional y el burocrático, pero que también podría integrar el estudio de las percepciones, las emociones, así como la metodología de análisis de redes sociales, por citar algunos ejemplos. En el caso de la desconcentración desde el estudio histórico de la política exterior de las grandes potencias, la agenda de investigación del realismo neoclásico se encuentra con el desafío de explicar los recientes desarrollos del orden mundial multipolar en el que se viene dando una redistribución del poderío y la emergencia de regiones como realidades geopolíticas que demandan más atención por derecho propio. Así, el futuro de la agenda realista neoclásica se encuentra en la exploración metodológica y en el análisis contemporáneo de las regiones y potencias emergentes integradas a un sistema global caracterizado por la difusión de poder.

El avance de una agenda como la sucintamente descrita tiene como rasgo central el seguimiento de la realidad internacional de conformidad con las transformaciones que desde hace pocas décadas viene experimentando el orden mundial. El realismo neoclásico no obliga a decidir entre enfocarse en la estructura o en los agentes, superando así la dicotomía entre estructuralistas y reflectivistas. Pero sí compele a una conexión de métodos entre distintos niveles de análisis, dejando expuestos a los EE.II. y rescatando 
la necesidad de compartir elementos analíticos de la política comparada, en especial en la conducta de las instituciones formales e informales, fuerte histórico de la CP. Así, si el realismo neoclásico ha de posibilitar alguna reunificación disciplinaria o al menos una reaproximación, ella está sujeta, a su vez, a la voluntad de los investigadores en seguirle el pulso a las regiones en su mutua interacción con un mundo multipolar.

\section{CONCLUSIÓN}

Los celos y resistencias disciplinarias son habituales en las ciencias sociales debido a los viejos complejos de legitimación científica positiva respecto a las ciencias nomotéticas y la posibilidad de interacción entre los objetos de estudio de las idiográficas. El florecimiento de subdisciplinas especializadas tiene la virtud de brindar crecientes oportunidades para la comprensión y explicación de fenómenos y acontecimientos específicos y algunas veces novedosos, siendo susceptibles de ser incorporadas como tesis de utilidad para el conjunto de las ciencias del ser humano y su sociedad. Pero ese florecimiento, si quiere responder al interés del conocimiento y al prestigio de las ciencias sociales, debe ajustarse a parámetros en la definición de objeto. Allí los EE.II. manifiestan un problema cuando se les pretende asumir como autónomos a la CP. Las corrientes teóricas asociadas al movimiento intelectual reflectivista dentro de los EE.II. son una manifestación en los avances por quebrar dichos complejos cientificistas en el sentido nomotético. No obstante, la lógica de la inferencia causal sigue teniendo un espacio destacado en las investigaciones politológicas en general.

Fueron expuestos los rasgos generales de la teoría del realismo neoclásico como una explicación de la política exterior que incorpora como variable interviniente aspectos de política interna, sobre todo en lo referido a las capacidades que tienen los grupos de poder para incluir sus preferencias en la agenda diplomática y, muy especialmente, el poder del Estado para extraer y movilizar recursos nacionales en procura del proyecto político dominante. Se aleja así el realismo neoclásico de los extremos, representados en las explicaciones estructuralistas del neorrealismo y por aquellas de origen interno como lo plantea el constructivismo social. Pero más importante aun, reafirma la centralidad del Estado en el análisis de política exterior en un mundo multipolar, reivindicando así el objeto común de estudio de la CP y los EE.II.

Las posibilidades reales de reunificación podrían asociarse a un potencial dominio del realismo neoclásico en los EE.II., siendo esto una condición necesaria pero no suficiente para lograr que estos se asuman indiscutiblemente como parte de la CP. Este potencial efecto secundario del realismo neoclásico ha pasado inadvertido hasta ahora. Los argumentos para la autonomía de los EE.II. como disciplina social dentro de la gama de ciencias sociales se debilitan en la medida en que se expone el objeto de estudio común, y este cobra mayor relevancia debido a la polaridad internacional y sus dinámicas de mayor autonomía y compromiso selectivo.

No obstante la lógica unidad disciplinaria y el potencial empuje del realismo neoclásico no escapa de nuestra atención que la autonomía que los EE.II. presentan en la práctica 
supone un obstáculo formidable por causa del efecto "dependencia del camino" en instituciones educativas. Concluimos que las razones de la autonomía de los EE.II. encuentran sentido en espacios académico-administrativos, pero que la fuerza de los hechos debilita el argumento de dicha autonomía. No parece haber estímulos para un cambio en la actual relación práctica de los EE.II. con la CP, en tanto se tiende a aceptar la unidad de lo político en la investigación científica y la política práctica, mas los costos de la unificación académico-administrativa resultarían elevados y las resistencias considerables. Además de existir un creciente interés por la diversificación de las ofertas de estudio y el interés que despierta la especialización temprana en un área de gran atractivo para jóvenes estudiantes que ingresan a las universidades procurando obtener un título de pregrado con inmediata proyección exterior.

\section{REFERENCIAS}

Allison, Graham y Philip Zelikow. 1999. Essence of Decision: Explaining the Cuban Missile Crisis. Nueva York: Longman.

Almond, Gabriel A. 2002. Ventures in Political Science: Narratives and Reflexions. Boulder: Lynner Rienner Publishers.

Aron, Raymond. 1963. "Paz y Guerra entre las Naciones". Madrid: Revista de Occidente.

Battistella, Dario. 2012. "Raymond Aron, Réaliste Néoclassique". Revue Études Internationales. XLIII (3): 371-388.

Barnett, Michael N. y Raymond Duvall. 2005. “Power in International Politics". International Organization 59 (1): 39-75.

Bouthoul, Gastón. 1984. Tratado sobre Polemología: Sociología de las Guerras. Madrid: Servicio de Publicaciones del E.M.E.

Brooks, Stephen G. 1997. “Dueling Realisms (Realism in International Relations)”. International Organization 51 (3): 445-477.

Bull, Headley. 2002. The Anarchical Society: A Study of Order in World Politics. Nueva York: Columbia University Press.

Caminal, Miguel. 1996. "Introducción: La Política como Ciencia". En Manual de Ciencia Política, editado por M. Caminal. Madrid: 17-36.

Carr, Edward H. 2004. La Crisis de los Veinte Años. Madrid: La Catarata.

Del Arenal, Celestino. 1981. "La Génesis de las Relaciones Internacionales como Disciplina Científica", en Revista de Estudios Internacionales 2 (4): 849-892.

Del Arenal, Celestino. 1990. Introducción a las Relaciones Internacionales. Madrid: Tecnos.

Doyle, Michael W. 1986. "Liberalism and World Politics". American Political Science Review 80 (4): 1151-1169.

Duroselle, Jean-Baptiste. 1998. Todo Imperio Perecerá. Teoría sobre las Relaciones Internacionales. México: Fondo de Cultura Económica.

Escudé, Carlos. 1992. Realismo Periférico: Bases Teóricas para una Nueva Política Exterior Argentina. Buenos Aires: Planeta.

Glaser, Charles L. 2010. Rational Theory of International Politics. The Logic of Competition and Cooperation. Princeton: Princeton University Press.

Glaser, Charles L. y Chaim Kaufmann. 1998. "What is the Offense-Defense Balance and Can We Measure it?". International Security 22 (4): 44-82.

Gilpin, Robert. 1987. The Political Economy of International Relations. Princeton: Princeton University Press.

Haluani, Makram. 1991. La Ciencia del Control Político. Caracas: Alfadil. 
Hellmann, Gunther. 2011. "International Relations as a Field of Study". En International Encyclopedia of Political Science, editado por B. Badie, D. Berg-Schlosser y L. Morlino. New York: Sage Publications, pp. 1297-1315.

Hoffmann, Stanley. 2001. "An American Social Science: International Relations". En International Relations-Still and American Social Science. Towards Diversity in International Thought, editado por R. M. A. Crawford y D. S. L. Jarvis. Albany: State University of New York Press, pp. 27-51.

Howard, Michael E. 1983. The Causes of Wars. Cambridge MA: Harvard University Press.

Jervis, Robert. 1976. Perception and Misperception in International Politics. Princeton: Princeton University Press.

Kennedy, Paul. 1987. The Rise and Fall of the Great Powers. Economic Change and Military Conflict from 1500 to 2000. Nueva York: Random House.

Kuper, Adam y Jessica Kuper (ed). 1996. The Social Science Encyclopedia. Londres: Taylor \& Francis.

Kurki, Milja. 2006. "Causes of a Divided Discipline: Rethinking the Concept of Cause in International Relations Theory". Review of International Studies 32 (2): 189-216.

Lebow, Richard Ned. 2003. The Tragic Vision of Politics. Ethics, Interests and Orders. Cambridge: Cambridge University Press.

Lobell, Steven E. 2009. "Threat assessment, the state, and foreign policy: a neoclassical realist model". En Neoclassical Realism, the State, and Foreign Policy, editado por S. E. Lobell, N. M. Ripsman y J. W. Taliaferro. Cambridge: Cambridge University Press, pp. 42-74.

Mandelbaum, Michael. 1998. "Is Major War Obsolete?" Survival 40 (4): 20-38.

Mansfield, Edward D. y Hellen V. Milner (ed).1997. The Political Economy of Regionalism. Nueva York: Columbia University Press.

Marsh, David y Paul Furlong. 2010. "A Skin, not a Sweater: Ontology and Epistemology in Political Science". En Theory and methods in Political Science, editado por D. Marsh y G. Stoker. Londres: Palgrave Macmillan, pp. 17-41.

May, Ernest R., Richard Rosecrane y Zara Steiner. 2010. History and Neorealism. Cambridge: Cambridge University Press.

Mearsheimer, John J. 1994. “The False Promise of International Institutions". International Security 19 (3): 5-49. Mearsheimer, John J. 2001. The Tragedy of Great Power Politics. Nueva York: W. W. Norton \& Company.

Morgenthau, Hans J. 1986. Política entre las Naciones. La Lucha por el Poder y la Paz. Buenos Aires: Grupo Editor Latinoamericano.

Moscovici, Serge e Ivana Markova. 2006. The Making of Modern Social Psychology. Cambridge: Polity Press.

Niebuhr, Reinhold. 1953. Christian Realism and Political Problems. Nueva York: Scribner.

Ostrom, Elinor.1998. "A Behavioral Approach to the Rational Choice Theory of Collective Action: Presidential Address, American Political Science Association, 1997".The American Political Science Review 92 (1): 1-22.

Pérez Toribio, Juan C. 2011. Retórica, argumentación y elección de teorías en T. S. Kuhn. Madrid: Editorial Académica Española.

Putnam, Robert D. 1988. "Diplomacy and Domestic Politics: The Logic of Two-Level Games". International Organization 42 (3): 427-460.

Quacquarelli Symonds. 2014a. "QS World University Rankings by Subject 2014 - Politics \& International Studies". [En línea] http://www.topuniversities.com/university-rankings/university-subjectrankings/2014/politics, [Consulta: 5.1.2014].

Quacquarelli Symonds. 2014b. "QS University Rankings: Latin America". [En línea] http://www. topuniversities.com/latin-american-rankings,[Consulta: 5.1.2014].

Rathbun, Brian C. 2007. "Uncertain about Uncertainty: Clarifying a Crucial Concept for International Relations Theory".International Studies Quarterly51 (3): 271-299.

Renouvin, Pierre. 1990. Historia de las Relaciones Internacionales. Vol. 2. Madrid: Akal.

Renouvin, Pierre y Jean-Baptiste Duroselle. 2000. Introducción a la Historia de las Relaciones Internacionales. México: Fondo de Cultura Económica.

Rose, Gideon. 1998. "Neoclassical Realism and Theories of Foreign Policy". World Politics 51 (1): 144-172.

Snyder, Glenn H. 2002. "Mearsheimer's World-Ofensive Realism and the Struggle for Security". International Security 27 (1): 149-173. 
Snyder, Jack. 1993. Myths of Empire: Domestic Politics and International Ambition. Ithaca: Cornell University Press.

Schweller, Randall L. 1997. “New Realist Research on Alliances: Refining, Not Refuting, Waltz's Balancing Proposition".The American Political Science Review 91 (4): 927-930.

Schweller, Randall L. 1998. Deadly Imbalances: Tripolarity and Hitler's Strategy of World Conquest. Nueva York: Columbia University Press.

Schweller, Randall L. 2004. "Unanswered Threats: A Neoclassical Realist Theory of Underbalancing”. International Security 29 (2): 159-201.

Spykman, Nicholas J. 2008. America's Strategy in World Politics: The United States and the Balance of Power. New Brunswick: Transaction Publisher.

Taliaferro, Jeffrey W. 2006. "State Building for Future Wars: Neoclassical Realism and the ResourceExtractive State".Security Studies 15 (16): 464-495.

Taliaferro, Jeffrey W., Steven E. Lobell y Norrin M. Ripsman. 2009. “Introduction: Neoclassical Realism, the State, and Foreign Policy".En Neoclassical Realism, the State, and Foreign Policy, editado por S. E. Lobell, N. M. Ripsman y J. W. Taliaferro. Cambridge: Cambridge University Press, pp. 1-41.

Truyol y Serra, Antonio. 2006. La Sociedad Internacional. Madrid: Alianza.

Tucker, Aviezer. 2012. "Sciences of Historical Tockens and Theoretical Types: History and the Social Sciences". En The Oxford Handbook of Philosophy of Social Science, editado por H. Kincaid. Nueva York: Oxford University Press, pp. 274-297.

Walt, Stephen M. 1998. "International Relations: One World, Many Theories". Foreign Policy 110: 29-32+34-46. Waltz, Kenneth N. 1954. Man, the State, and War. A Theoretical Analysis. Nueva York: Columbia University Press.

Waltz, Kenneth N. 1979. Theory of International Politics. Berkeley: University of California Press.

Waltz, Kenneth N. 1996. "International Politics is not Foreign Policy”. Security Studies 6 (1): 54-57.

Wendt, Alexander. 1987. "The Agent-Structure Problem in International Relations Theory". International Organization 41 (3): 335-370.

Wendt, Alexander. 1999. Social Theory of International Politics. Cambridge: Cambridge University Press.

Wight, Colin. 2002. "Philosophy of Social Science and International Relations". En Handbook of International Relations, editado por W. Carlnaes, T. Risse y B. A. Simmons. Londres: Sage, pp. 23-51.

Williams, Michael C. 2005. The Realist Tradition and the Limits of International Relations. Cambridge: Cambridge University Press.

Wohlforth, William C. 1993. The Elusive Balance: Power and Perceptions during the Cold War. Ithaca: Cornell University Press.

Zakaria, Fareed. 1998. From Wealth to Power: The Unusual Origins of America's World Role. Princeton: Princeton University Press.

Víctor M. Mijares es Profesor Asistente del Departamento de Ciencias Sociales de la Universidad Simón Bolívar, investigador del German Institute of Global and Area Studies y candidato a Doctor en Ciencia Política por la Universidad de Hamburgo. Sus intereses de investigación se centran en seguridad internacional, análisis de política exterior, economía política del petróleo y teoría de las relaciones internacionales. E-mail: vmijares@usb.ve / victor.mijares@giga.hamburg 
